\title{
Electrophoretic Studies of the Geographic Distribution of Yersinia pestis Protein Variants
}

\author{
B. W. HUDSON, T. J. QUAN, AND R. E. BAILEY \\ Vector-Borne Diseases Division, Center for Disease Control, Fort Collins, Colorado 80522
}

\begin{abstract}
Discontinuous electrophoresis techniques were used to analyze 161 Yersinia pestis isolates from Asia, Southeast Asia, Java, Madagascar, Africa, and North and South America. The application of similarity matrix analysis to electrophophoretic data from isolates of each geographic region led to the grouping of the isolates into 57 electropherotypes which, in turn, form 11 electropherotype groups representing major protein variants. Of the 161 isolates tested, only seven, belonging to two pairs of the 57 electropherotypes, could not be differentiated using the electrophoretic analysis and six biochemical tests. In addition, the distribution of certain of the 11 major electropherotype groups corresponds with known routes of dispersion of $Y$. pestis during the present pandemic.
\end{abstract}

Yersinia pestis isolates obtained after an outbreak of plague which occurred in Central Java in 1968 (3) were observed to possess an unusual distribution of protein when examined by discontinuous acrylamide gel electrophoresis techniques (8). Subsequent investigations with a number of $Y$. pestis cultures obtained from Java, the Republic of Vietnam, Burma, Yemen, Brazil, Bolivia, and the United States revealed quantitative differences which characterized isolates from certain geographic areas (10). In addition, $Y$. pestis cultures with electropherograms similar to the original Javanese cultures were found in collections from Brazil, Burma, and Yemen. This led to the speculation that such isolates might be of limited tropical or subtropical distribution. The number of cultures from these areas was not sufficient, however, to verify this observation. Additional results indicated that, in two locations, cultures collected a number of years apart were virtually identical. With the limited means of statistical evaluation available to the authors at that time, only a small number of geographic groups of plague isolates could be compared.

Since the second report in this series (10), a number of colleagues have suggested further evaluation of the utility of discontinuous gel electrophoresis for differentiating major protein variants of $Y$. pestis. As part of this effort, 161 $Y$. pestis- $Y$. pestoides cultures were compared with $8 Y$. enterocolitica and $10 Y$. pseudotuberculosis cultures (9). Although $Y$. pestis, $Y$. enterocolitica, and $Y$. pseudotuberculosis could be easily identified on the basis of qualitative electrophoretic characters, no qualitative characteristics useful in identifying the various varieties and subspecies of the $Y$. pestis- $Y$. pestoides complex were found. We have, therefore, examined the $Y$. pestis- $Y$. pestoides complex with the view of describing the major quantitative protein variants present in the sample, as well as the geographic distribution of such variants.

\section{MATERIALS AND METHODS}

Bacterial isolates were selected to include as varied a sample as possible from each geographic region studied. Owing to the lack of correlation between electrophoretic characteristics and host data in previous studies (10), only locality and date of isolation, where available, are included on the following list. Other data will be supplied on request.

United States. California, 1966: CS118T, CS283, CS443, CS445, CS338, CS339, CS446, CS617, CS22127, CS619, CS863. California, 1968: T29. California, 1970: 702596. California, 1971: 715037, 715038, 715039, 715006. California, 1972: 723064T. Colorado, 1963: 63CP55. Utah, 1965: 65UT24. New Mexico, 1963: S.D.

Bolivia. 1965: Aguilar. 1969: 69-62-1.

Peru. Ancash, 1955: Peru 8. Ancash, 1957: Peru 22. Puira, 1960: Peru 65, 68, 71, 81, 88, 89, 92, 94, 100. Puira, 1961: Peru 111. Puira, 1968: Peru 191, 192. Puira, 1971: Peru 196, 197.

Ecuador. Date and locality unknown. Ecuador 1, 2, 3, 4, 5, 7, 8, 13.

Brazil. Pernambuco, Exú, 1966-67: Exú 1, 2, 5, 7, $8,9,10,11,12,13,15,16,18,19,20$. Pernambuco, Triunfo, 1966-67: Exú 14, 17.

Yemen. 1968: PYH-1, PYH-2.

Iran. Kurdistan, 1962-64; PKH-9, PKH-10, PKR133, PKR-159.

Burma. Myingyan, 1969: 14-33, -34, -35, -36; place and date unknown, $14-37,-38,-39,-40,-41,-42$, $-43,-44,-45$. 
Nepal. 1967-69: Nepal 514, 515, 516, Rhamgiri, T464.

Vietnam. Haumghia, 1970: 16-37, -66, 17-57, -65; Komtum, 1969-70: 16-34, -53, 17-87.

India. Barabanki, 1955: PB-6, PB-43.

Manchuria. Chiyencheon, 1942-43: Chiy. Nicholisk, Nich. 41, 51. Harbin, Har. 35, 37.

Union of Soviet Socialist Republics. Dates and localities unknown. YP35, -36, -37, -38, -39, -70, $-76,-78,-91$, G. (Culture YP91 was originally submitted as a U.S.S.R. isolate; after this study was completed, we were informed that the isolate was of Vietnamese origin.)

Kenya. Nairobi, 1965: Nairobi.

Republic of South Africa. Orange Free State, 1950: V13/50. Cape Province, 1950: V201/50. Cape Province, 1953: F607/53. Cape Province, 1967: H4/67. Ovamboland, 1966: H19/66, F361/66, H21/66, H4/66. Ovamboland 1968: H6/68, H19/68, H3/68. Lesotho, 1968: F340/68, D16/68, F308/68.

Madagascar. 1951: Mad 21, $103_{\mathrm{a}}, 107,108,109_{3}$, $110,111_{\mathrm{a}}, 112,115,115_{3}, 117_{\mathrm{a}}, 128, \mathrm{H}_{3}, \mathrm{RP}$.

Java. Central Java, Bojolali, 1957: Java 4, 5, 6, 7. Central Java, Bojolali, 1968: J770, 777, E548, 551, 560, Muradi, Semen, Sutar, Tumini. Surakarta, 1957: Java 1, 2, 3, 8, 9, 10, 11.

Y. pestis biochemical variants. A series of selected laboratory variants of the KIM-10 parent stock. KIM-10, KIM-10 A+, KIM-10 P-, KIM-10 RHA + P-, KIM-10 VW-P-, KIM 10 F-P-.

Growth and processing of bacteria. $Y$. pestis cultures were grown in beef neart infusion broth at 28 C. Roux bottles containing blood agar base (BBL, pH 7.3) were then inoculated from the 24-h broth cultures and incubated for 3 days at $28 \mathrm{C}$. Roux bottle cultures were harvested by using $0.85 \%$ sodium chloride solution and were killed by adding the resulting suspension to 4 volumes of cold $(-20 \mathrm{C}$ ) reagent grade acetone. After the suspensions had been held at room temperature overnight, the sediment was washed two times with cold acetone; excess acetone was removed by using vacuum, and the powders were stored at $-20 \mathrm{C}$.

Discontinuous acrylamide gel electrophoresis. Discontinuous acrylamide gel electrophoresis and photometric measurement techniques were performed by procedures previously presented $(9,10)$. All apparatus used was acquired from the Canalco Industrial Corp. Aqueous extracts of acetone powders were prepared and analyzed by using discontinuous electrophoresis in $7 \%$ acrylamide gels. Resulting electropherograms were stained with amido black. The relative optical densities of the various stained bands which resulted were then measured by using a scanning microdensitometer. To eliminate variation caused by different dye batches, we used the same dye solution throughout the experiment.

As previously reported (9), discontinuous acrylamide gel electropherograms of $Y$. pestis and $Y$. pestoides contain from 18 to 25 visible protein bands. The nine major protein bands found in all $Y$. pestis isolates were as previously diagramed $(9,10)$. Bands in figures in this report are numbered in accordance with the system given in previous publications.
Statistical procedures. For each $Y$. pestis electropherogram, the optical densities for nine selected protein bands were measured. The relative optical density (ROD) for band $i(i=1, \ldots, 9)$ was defined to be:

$$
\operatorname{ROD}_{i}=\frac{x_{i}}{\sum_{2=1}^{9} x_{i}}
$$

where $x_{1}=$ the optical density for protein band $i$.

With the aid of the Center for Disease Control's IBM 370/145 computer and programs written by G. D. Waters of the computer staff, numerical taxonomic methods were applied to the specimens. First, a similarity matrix consisting of sample product-moment correlation coefficients ( $r$ ) for all possible pairs of specimens belonging to the subgroup being examined was constructed. These similarity coefficients were based upon the nine measurements of relative optical densities made on each specimen. Then, a clustering algorithm, to cluster by average similarity, was performed on the similarity matrix, and the results of the process were graphically displayed as dendograms. Lee (12), Lee and Tauraso (13), and Dowdle et al. (6) used a similar method to clarify relationships among multiple strains of influenza virus. (The computer programs for clustering by average similarity are available from the Center for Disease Control, G. D. Waters, Computer Systems Branch, Atlanta, Ga. 30333.)

To assess the effectiveness of our grouping scheme for various grouping configurations, we used a statistical procedure developed by Johnson (11) as the measure of closeness in determining whether responses were relatively homogenous within previously determined subgroups. Also, a detailed description of this procedure is in press $(\mathrm{P}$. W. Mielke, K. J. Berry, and E. S. Johnson, Communications in Statistics).

Bacteriology: biochemical tests. All cultures used in this study were received as confirmed isolates of $Y$. pestis or were confirmed as such in this laboratory by using recommended methods (1). Standard identification tests included: microscopic and macroscopic morphology, staining reactions, sensitivity to $Y$. pestis-specific bacteriophage, presence of specific virulence antigens, pathogenicity for laboraory animals, and serological reactions. Other bacteriological identification tests giving consistent results for $Y$. pestis cultures have included acid production from glucose and mannitol and lack of acid production from lactose, melibiose, or sucrose. Additionally, all $Y$. pestis strains have been urease negative, indole negative, $\mathrm{H}_{2} \mathrm{~S}$ negative, nonhemolytic, nonmotile, and have produced no change in litmus milk.

Tests for acid production from glycerol, rhamnose, arabinose, maltose, and xylose and for reduction of nitrate to nitrite have had variable results for $Y$. pestis isolates from different areas and were included in this study because of their potential value in characterizing the cultures.

Tubes containing nitrate broth $\left(1 \% \mathrm{KNO}_{3}\right.$ in nutrient broth) or $1 \%$ carbohydrate (glycerol, rham- 
nose, arabinose, maltose, or xylose) in purple broth base (BBL, $\mathrm{pH}$ 6.8) were inoculated with about 0.1 $\mathrm{ml}$ of $20-\mathrm{h}$ broth cultures of the various $Y$. pestis isolates and incubated at $37 \mathrm{C}$ (1). Tests for the reduction of nitrate to nitrite were performed at $\mathbf{4 8}$ h (4). The carbohydrate solutions were examined daily for 7 days.

\section{RESULTS}

Analysis of the effects of laboratory selection on disc gel characteristics. For the analysis of effects of laboratory selection on disc gel characteristics, we used selected laboratory variants of the $Y$. pestis KIM-10 strain. Densitometric profiles of disc gel electropherograms prepared from the KIM-10 series are shown in Fig. 1. Arabinose $(\mathrm{A}+)$ and rhamnose $(\mathrm{RHA}+)$ fermentation, as well as absence of pigment $(\mathrm{P}-)$, virulence antigens $(\mathrm{VW}-)$, and envelope antigen $(F-)$, are considered to be characteristic of marked variation in plague isolates obtained from natural surroundings. Marked differences in protein distribution are evidenced solely by the reduced amplitude of bands $6,7,8$, and 9 in the arabinose-positive variant (KIM$10 \mathrm{~A}+$ ). A dendrogram resulting from the numerical taxonomic analysis of the six specimens is shown in Fig. 2. A similarity coefficient of 1.00 would indicate identical values for the specimens or groups being compared. Branching points reflect mean similarity matrix coefficients for all members of the group to the left of the branching point in question. The majority of the KIM-10 series of cultures, with the exception of KIM-10A+, are very similar ( $r>$ 0.98 ) with regard to the relative quantities of protein present in each of the nine disc gel protein bands selected for comparison. The average similarity coefficient $(r)$ for the whole KIM-10 group was 0.967 .

Electrophoretic studies of geographic variation of $Y$. pestis. Duplicate electropherograms were prepared for each of the $161 Y$. pestis isolates studied in this phase of the experiment. As an estimate of expected variation caused by sampling and analytical procedures, similarity coefficients were calculated for each pair of duplicate electropherograms. The mean value of the 161 coefficients was $r=0.994$ with standard deviation $\mathrm{s}=0.0077$. We assumed 2 standard deviations $(r \geq 0.978)$ to be an approximation of the experimental variation expected for strains or groups of isolates not distinguishable by electrophoretic comparison. A value of four standard deviations $(r \geq 0.963)$ was arbitrarily chosen as an estimate of expected variation of a family of related isolates within a specified geographic region. This value is consistent with that obtained from the KIM10 variant series, $\mathrm{r}=0.967$.

Electrophoretic data for $Y$. pestis isolates were combined and analyzed separately in $\mathbf{1 0}$ geographic groupings consisting of isolates from Brazil, Southeast Asia (India, Burma, Nepal, Vietnam), Asia (U.S.S.R., Manchuria), Africa (Kenya, Republic of South Africa), Java, Yemen, Iran, South America (Bolivia, Peru, Ecuador), North America, and Madagascar. For each isolate, the duplicate measurements of the relative optical densities for each of the nine selected protein bands were averaged before numerical taxonomic analysis. Groups of indistinguishable isolates $(r \geq 0.978)$ were designated strains, and similar groups $(r>0.963)$ of such strains obtained within one of the geographic regions were identified as electropherotypes. Specimen dendrograms from which such groupings were obtained are shown in Fig. 3.

For the 10 geographic regions analyzed, a total of 58 electropherotypes were obtained originally (Tables 1,2 ). To compare the similarities between these 58 electropherotypes, we used the average relative optical density measurements (Table 1) for specimens within each of the electropherotypes (i.e., nine-dimensional mean vectors) to construct a similarity matrix dendrogram. The resulting dendrogram showing similarities between mean values obtained for electropherotypes from all the geographic regions sampled is shown in Fig. 4. The dendrogram reveals 11 major protein variants or groupings of $Y$. pestis electropherotypes. These have been labeled A through $\mathrm{K}$ in Fig. 5, which presents densitometric recordings of representative members of the larger groups $\mathrm{A}$ to $\mathrm{G}$. Groups $\mathrm{H}$ to $\mathrm{J}$ are represented by only one $Y$. pestis isolate each and, although they were confirmed $Y$. pestis isolates as discussed in Materials and Methods, they may be single aberrant cultures. For this reason we have illustrated only $J$ and $K$ in the figure. Only certain groups possess striking differences on visual inspection; notable in this regard are the representatives of groups A, B, E, J, and K.

In addition to the usual bacteriological tests recommended for identification of $Y$. pestis, six additional biochemical tests were performed on each isolate. Glycerol $(\mathrm{G}+)$, nitrate $(\mathrm{N}+)$, and rhamnose (RHA + ) utilization have been employed to characterize major biotypes or subspecies of $Y$. pestis $(5,16)$. Seven of the eight potential combinations of these three characteristics were present in the sample of 161 isolates tested. Three additional bacteriological tests, usually considered to be of questionable taxonomic value, were utilized for 


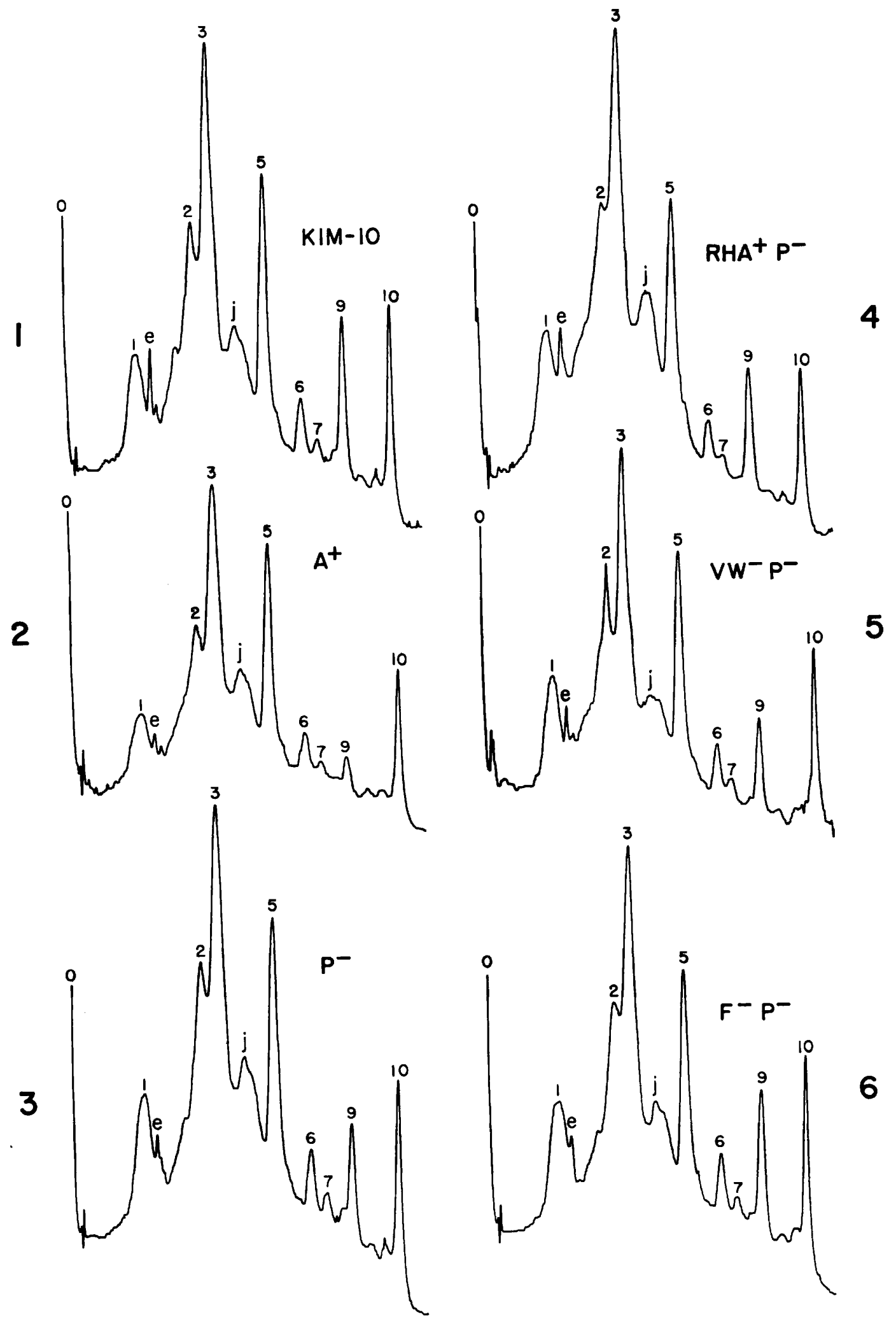

Fig. 1. Densitometric profiles of discontinuous acrylamide gel electropherograms of biochemical variants of Yersinia pestis culture KIM-10. Abbreviations: A, arabinose; $P$, pigment formation; $R H A$, rhamnose; $V W$, virulence antigens; $F$, envelope antigen (fraction 1 ). 


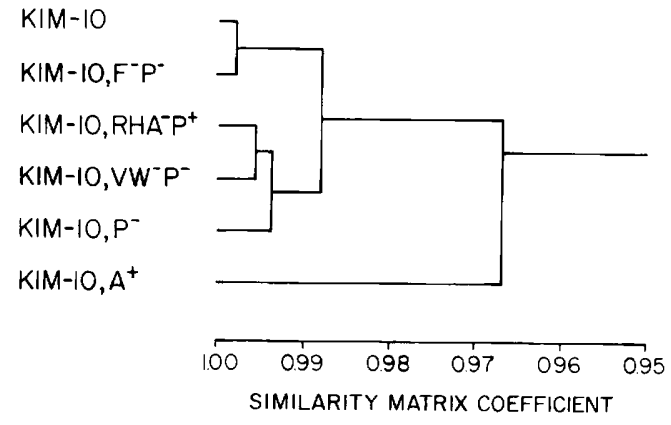

FIG. 2. Similarity matrix dendrogram showing relative similarities of a series of selected Yersinia pestis biochemical variants. Abbreviations: KIM-10, parent culture; $A+$, arabinose positive; $R H A+$, rhamnose positive; $P-$, pigment negative; $V W-$, lacking $V$ and $W$ antigen; $F-$, lacking fraction 1 envelope antigen. A similarity coefficient of 1.00 indicates identical values for specimens or groups being compared. Branching points reflect mean simi- minor biotype characterization. These were selected on the basis of earlier studies which demonstrated their potential value for characterizing isolates previously grouped by electrophoretic characteristics (10). These were acid production from arabinose $(\mathbf{A}+)$, maltose $(\mathbf{M}+)$, and xylose $(\mathrm{X}+)$.

Because of the complexity of the biotype categories available from these six tests, the 64 potential biotypes have been coded by the scheme presented in Table 3 which also presents the named subspecific designations of the $Y$. pestis- $Y$. pestoides complex. A designation such as $\alpha$-e, therefore, represents an isolate characterized as $\mathrm{G}+\mathrm{N}+\mathrm{RHA}+\mathrm{A}-\mathrm{M}+\mathrm{X}+$. In other terminologies, this might be designated as an arabinose negative $\left(\mathrm{A}^{-}\right)$isolate of the

larity matrix coefficients for all members of the group to the left of the branching point in question.

\begin{tabular}{|c|c|c|}
\hline \\
\hline \multicolumn{3}{|l|}{$\frac{A \text { JAVA }}{\text { AREA }}$} \\
\hline $\begin{array}{l}\text { BOJOLALI } \\
\text { BOJOLALI }\end{array}$ & \begin{tabular}{|l|}
1968 \\
1968
\end{tabular} & $\begin{array}{l}\text { MURADI } \\
\text { SUTAR }\end{array}$ \\
\hline BOJOLALI & 1968 & SEMEN \\
\hline LI & 1968 & TUM \\
\hline BOso & 1968 & E 56 \\
\hline BOJOL & 1968 & JAVA \\
\hline BOJOLALI & 196 & JAVA 7 \\
\hline BOJOLALI & 1968 & E548 \\
\hline & 8 & JAVA \\
\hline 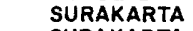 & $19:$ & JAV \\
\hline $\begin{array}{l}\text { SURA } \\
\text { BOJC }\end{array}$ & $\begin{array}{l}1957 \\
1957\end{array}$ & $\begin{array}{l}\text { JAVA II } \\
\text { JAVA } 7\end{array}$ \\
\hline BOJOLALI & 1957 & JAVA 5 \\
\hline $80 \mathrm{se}$ & 1957 & $\begin{array}{l}\text { JAVA } 4 \\
\text { JAVA }\end{array}$ \\
\hline & $\mid$ & JAVA 9 \\
\hline & 1957 & JAVA IO \\
\hline & 1957 & JAV \\
\hline st & |1957| & JAVA \\
\hline
\end{tabular}

$$
\text { 10.0 }
$$

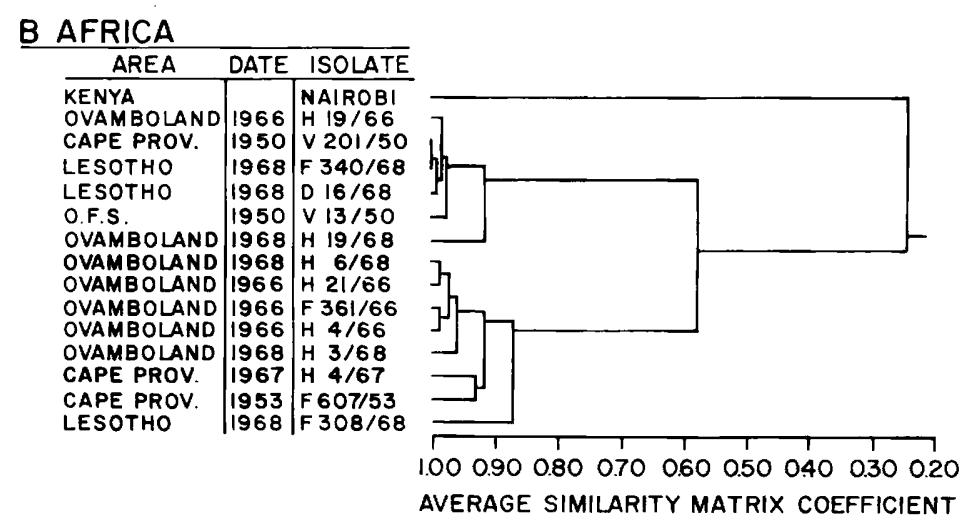

FIG. 3. Similarity matrix dendrograms of Javanese and African Yersinia pestis isolates. A similarity coefficient of 1.00 indicates identical values for isolates or groups of isolates being compared. Branching points reflect mean similarity matrix coefficients for all members of the group to the left of the branching point in question. 


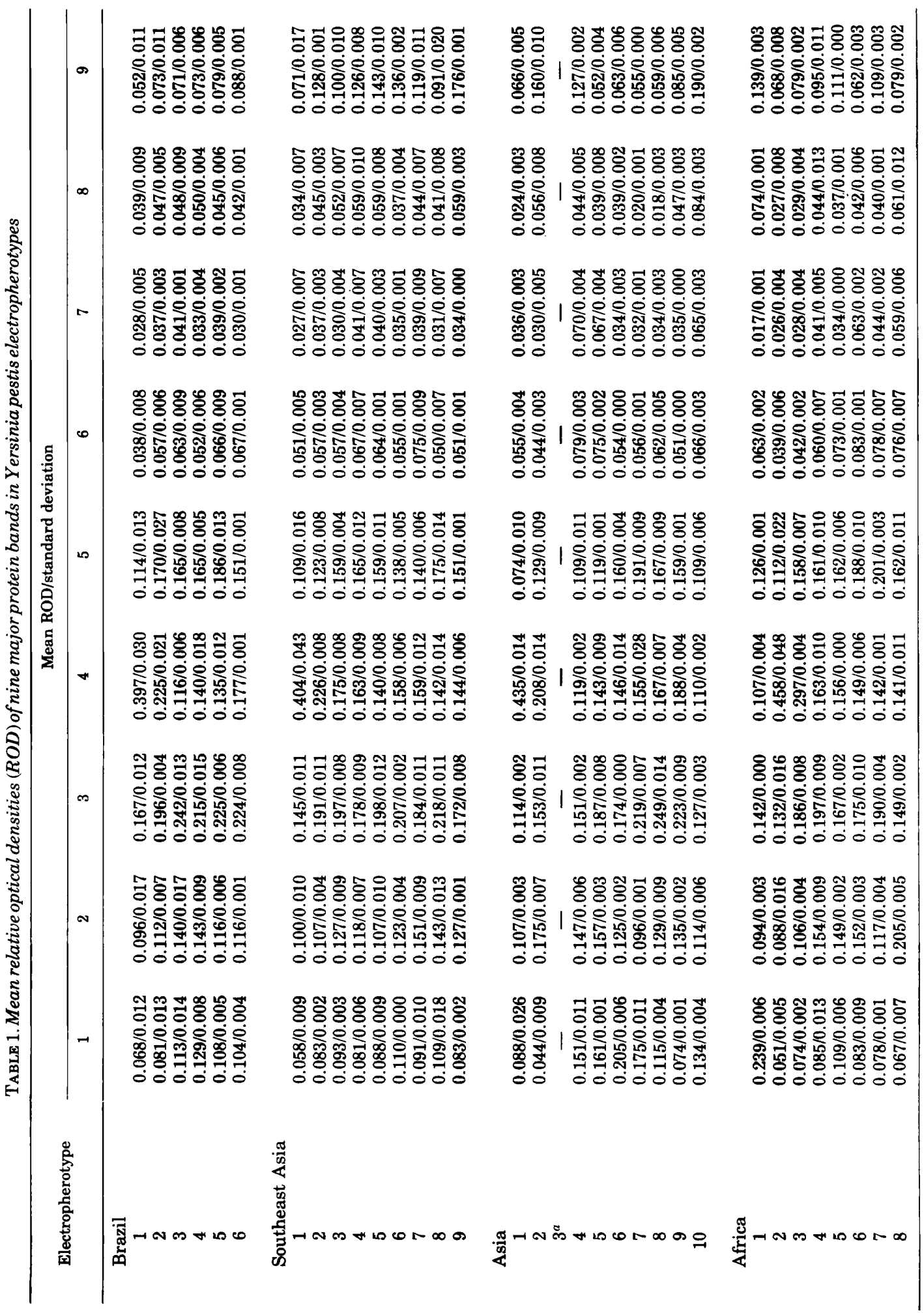




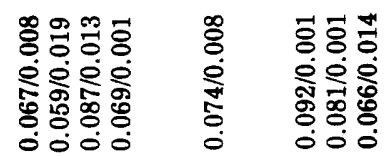

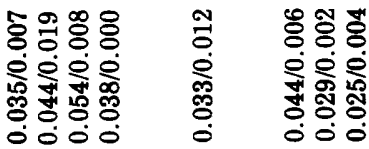

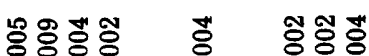

ㅇํㅇㅇㅛ

ํㅇㅇ융 낭

영영

ํํㅇㅎㅇㅇㅎㅇ

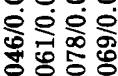

$0 \circ 00$

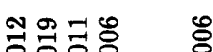

ㅇํㅇํㅇ

츤 온

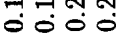

్ํㅇ 융용효

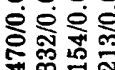

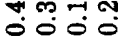

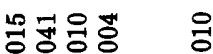

웅요을

象蛋题

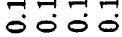

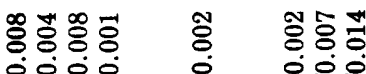

웅요

ชํㅇㅇㅇㅇㅇㅇ

Oㅇㅇㅇ

ஜㅎㅇ융

응용ㅇㅇㅇ

눙영융

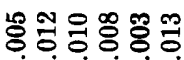

웅ㅇㅇㅇㅇ

긍융동형

○ं0்

궁영형ㄷㅎㅇ훙응

iेंश்ं

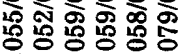

ஸें

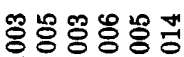

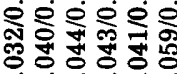

○ं0ல்

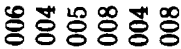

ஸेंशंधि

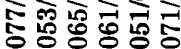

○ं

형킁경홍홍영

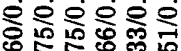

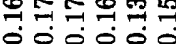

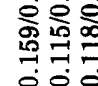

동횽홍

ㅇํㅇ요용

ல்

농횽용

ํํㅇํํ

경웡

언즁

ச்

컹동

ㅇํㅇํํ

옹 익 오

둥.
긍쿵융응 궁융

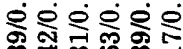

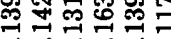

○ं0்

켱융응응영 옹

영ํํㅇํํ용

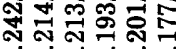

ㅇํㅇㅇํㅇ융 궁

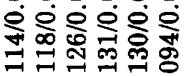

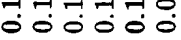

눙양응음혛

ํํㅇํㅇํㅇํㅇ

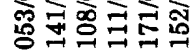

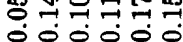

○ंல்
영영영 융ㅇㅇㅇㅇㅇㅇ

웅요옹요

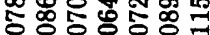

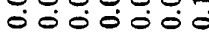

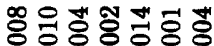

ㅇํㄹ요용ํํ

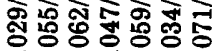

¿ं0்

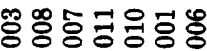

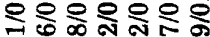

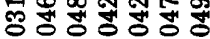

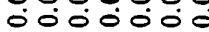

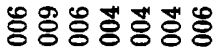

웅효용요

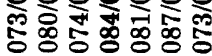

ல

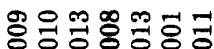

옥영융ํㅇ용

ํำ

ல்ல்ல்

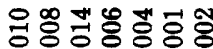

ㅇํㅇ요용

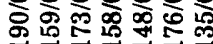

0ं0்

청경음음형형영

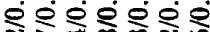

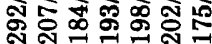

ஸें

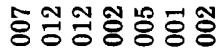

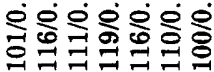

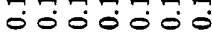

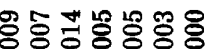

ㅇํㅇ용요용ㅇㅇ

भंग

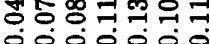

응 꼉 웅홍

운오오

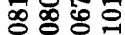

0000

ซ๊

சं்

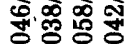

0000

ㅇํㅇㅎㅇㅇㅇㅇㅇㅇㅇ

웅요

ฟั용 낭

0000

능융ㄴㅇㅇㄴㅇㅇ

ํํㅇํํ

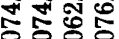

○ं 000

영응형융

응응용

空

○ं०்

응 동융형

i่

촘

0000

옹응형ㅎㅇ

옹옹옹ㅇㅇ

옹 호용

엉ㅇㅇ

긍 ㅎํㅇํํㅇํํ

웅옹

켝 궁

궁ㅇㅇㅇ

눙 웡 \%ㅇㅇㅇ

ㅇํㅇํㅇ

o

웅둥

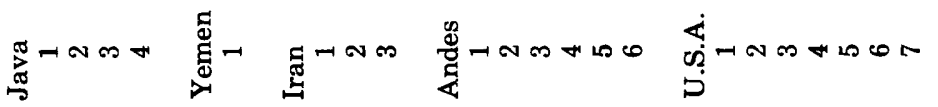

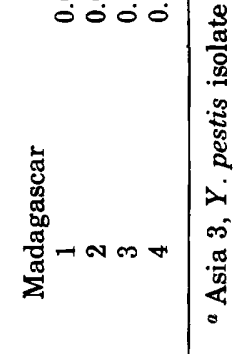


TABLE 2. Characterization of 161 Yersinia pestis isolates by electropherotype and biotype

\begin{tabular}{|c|c|c|c|c|c|}
\hline \multirow{2}{*}{ Origin } & \multicolumn{4}{|c|}{ Electropherotype } & \multirow{2}{*}{ Biotypes $^{c}$ and isolate designations } \\
\hline & Name & $\mathbf{r}^{a}$ & $\mathrm{n}^{b}$ & Group & \\
\hline \multirow[t]{6}{*}{ Brazil, Pernambuco } & Brazil 1 & 0.984 & 6 & $\mathbf{A}$ & $\begin{array}{l}\zeta-\mathrm{e}, \text { Exú } 7,8,11,13 \\
\zeta-g, \text { Exú } 5,10\end{array}$ \\
\hline & Brazil 2 & 0.963 & 2 & C & 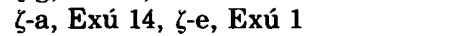 \\
\hline & Brazil 3 & 0.970 & 3 & $\mathrm{D}$ & $\zeta$-e, Exú 15, 18, 19 \\
\hline & Brazil 4 & 0.987 & 2 & D & $\zeta$-e, Exú $2, \zeta-g$, Exú 9 \\
\hline & Brazil 5 & 0.981 & 3 & $\mathbf{D}$ & $\zeta$-e, Exú $12, \zeta$-g, Exú 16, 17 \\
\hline & Brazil 6 & - & 1 & $\mathrm{D}$ & $\zeta$-g, Exú 20 \\
\hline \multicolumn{6}{|l|}{ Southeast Asia } \\
\hline India, Barabanki & SEA 1 & 0.984 & 5 & A & $\zeta-\mathrm{a}, \mathrm{PB}-6,-43$ \\
\hline Burma & SEA 1 & & & & $\zeta-\mathrm{a}, 14-41,-44,-45$ \\
\hline Burma & SEA 2 & - & 1 & B & $\zeta-a, 14-35$ \\
\hline Nepal & SEA 3 & 0.986 & 2 & $\mathrm{D}$ & $\zeta$-f, Nepal 514 \\
\hline Vietnam & SEA 3 & & & & $\zeta-b, 17-87$ \\
\hline Burma & SEA 4 & 0.977 & 5 & $\mathbf{F}$ & $\theta-\mathrm{a}, 14-40, \zeta-\mathrm{a}, 14-34,-36,-39$ \\
\hline Vietnam & SEA 4 & & & & $\zeta-\mathrm{a}, 17-65$ \\
\hline Vietnam & SEA 5 & 0.972 & 3 & $\mathrm{~F}$ & $\zeta-a, 16-37,-53,-66$ \\
\hline Vietnam & SEA 6 & - & 1 & $\mathbf{F}$ & $\zeta-\mathrm{a}, 16-34$ \\
\hline Burma & SEA 7 & 0.963 & 5 & $\mathbf{F}$ & $\begin{array}{l}\zeta-\mathrm{a}, 14-33,-37,-42 ; \theta-\mathrm{a}, 14-38, \epsilon-\mathrm{a} \\
\quad 14-43\end{array}$ \\
\hline Nepal & SEA 8 & 0.966 & 4 & D & $\begin{array}{l}\delta \text {-g, Nepal 516; } \delta \text {-h, Rhamgiri; } \beta \text {-g, } \\
\text { Nepal 515, T464 }\end{array}$ \\
\hline Vietnam & SEA 9 & - & 1 & $\mathbf{F}$ & $\zeta-c, 17-57$ \\
\hline \multicolumn{6}{|l|}{ Asia } \\
\hline Manchuria, Chiyencheon & Asia 1 & 0.990 & 2 & A & $\alpha-a$, Chiy. \\
\hline U.S.S.R. & Asia 1 & & & & $\zeta-\mathrm{a}, \mathrm{YP}-78$ \\
\hline U.S.S.R. & Asia 2 & 0.988 & 3 & $\mathrm{~B}$ & $\gamma-\mathrm{a}, \mathrm{YP}-36 ; \gamma-\mathrm{e}, \mathrm{YP}-35,-37$ \\
\hline U.S.S.R. & Asia $3^{d}$ & - & 1 & $\mathbf{F}$ & e-a, YP-91 \\
\hline Manchuria, Nicholisk & Asia 4 & - & 1 & I & $\gamma$-a, Nich. 41 \\
\hline Manchuria, Harbin & Asia 5 & - & 1 & $\mathrm{G}$ & $\beta$-a, Har. 37 \\
\hline Manchuria, Nicholisk & Asia 6 & - & 1 & $\mathrm{G}$ & $\alpha-b$, Nich. 51 \\
\hline U.S.S.R & Asia 7 & - & 1 & $\mathrm{G}$ & $\alpha-\mathrm{a}, \mathrm{YP}-70$ \\
\hline U.S.S.R. & Asia 8 & 0.989 & 3 & $\mathrm{D}$ & $\alpha-\mathrm{a}, \mathrm{YP}-38,-39,-\mathrm{G}$ \\
\hline U.S.S.R. & Asia 9 & - & 1 & D & $\alpha$-a, YP-76 \\
\hline Manchuria, Harbin & Asia 10 & - & 1 & $\mathrm{~K}$ & $\delta$-a, Har. 35 \\
\hline \multicolumn{6}{|l|}{ Africa } \\
\hline Kenya, Nairobi & Africa 1 & - & 1 & $\mathrm{~J}$ & $\beta-\mathrm{a}$, Nairobi \\
\hline $\begin{array}{l}\text { Republic South Africa } \\
\text { O.F.S. }\end{array}$ & Africa 2 & 0.983 & 5 & A & $\zeta-\mathrm{h}, \mathrm{V} 13 / 50$ \\
\hline Cape Province & Africa 2 & & & A & $\zeta-\mathrm{g}, \mathrm{V} 201 / 50$ \\
\hline Ovamboland & Africa 2 & & & $\mathrm{~A}$ & $\zeta$-c, H19/66 \\
\hline Lesotho & Africa 2 & & & A & $\zeta-\mathrm{a}, \mathrm{F} 340 / 68, \mathrm{D} 16 / 68$ \\
\hline Ovamboland & Africa 3 & - & 1 & $A$ & $\zeta$-c, H19/68 \\
\hline Ovamboland & Africa 4 & 0.975 & 4 & $\mathrm{D}$ & $\zeta$-c, F361/66, H6/68, H21/66; -d, H4/66 \\
\hline Ovamboland & Africa 5 & - & 1 & $\mathrm{~F}$ & $\zeta-\mathrm{c}, \mathrm{H} 3 / 68$ \\
\hline Cape Prov. & Africa 6 & - & 1 & $\mathrm{C}$ & $\zeta-\mathrm{a}, \mathrm{H} 4 / 67$ \\
\hline Cape Prov. & Africa 7 & 一 & 1 & $\mathrm{C}$ & $\zeta-\mathrm{g}, \mathrm{F} 607 / 53$ \\
\hline Lesotho & Africa 8 & - & 1 & $\mathrm{H}$ & $\zeta-\mathrm{a}, \mathrm{F} 308 / 68$ \\
\hline \multicolumn{6}{|l|}{ Java } \\
\hline Surakarta & Java 1 & 0.992 & 13 & A & $\zeta$-e, Java 2, 11 \\
\hline Bojolali & Java 1 & & & & $\begin{array}{l}\zeta \text {-e, Java } 6,7,770,777 \text { E } 548,551,560 \\
\text { Muradi, Sutar, Semen, Tumini }\end{array}$ \\
\hline Bojolali & Java 2 & 0.965 & 2 & A & $\zeta$-e, Java 4, 5 \\
\hline Surakarta & Java 3 & 0.977 & 4 & C & $\zeta$-e, Java $1,3,9,10$ \\
\hline Surakarta & Java 4 & - & 1 & C & $\zeta$-e, Java 8 \\
\hline Yemen & Yemen 1 & 0.981 & 2 & A & $\delta$-a, PYH-1, -2 \\
\hline
\end{tabular}


TABLE 2-Continued

\begin{tabular}{|c|c|c|c|c|c|}
\hline \multirow{2}{*}{ Origin } & \multicolumn{4}{|c|}{ Electropherotype } & \multirow{2}{*}{ Biotypes $^{c}$ and isolate designations } \\
\hline & Name & $r^{a}$ & $\mathrm{n}^{b}$ & Group & \\
\hline \multicolumn{6}{|l|}{ Iran } \\
\hline \multirow[t]{3}{*}{ Kurdistan } & Iran 1 & - & 1 & $\mathrm{D}$ & $\delta-\mathbf{a}$, PKH-9 \\
\hline & $\operatorname{Iran} 2$ & - & 1 & $\mathrm{G}$ & $\beta-c$, PKR-159 \\
\hline & Iran 3 & 0.972 & 2 & G & $\delta$-a, PKH-10, -133 \\
\hline \multicolumn{6}{|l|}{ South America } \\
\hline Bolivia & Andes 1 & 0.990 & 2 & $\mathbf{E}$ & $\zeta-\mathrm{a}, 69-62-1 ; \zeta-\mathrm{e}$, Aguilar \\
\hline Peru & Andes 2 & 0.980 & 8 & G & $\begin{array}{l}\zeta \text {-e, Peru } 68 ; \zeta \text {-a, Peru } 8,71,81,89, \\
94,111,191\end{array}$ \\
\hline Peru & Andes 3 & 0.969 & 8 & $\mathrm{D}$ & $\zeta$-e, Peru $22 ; \zeta$-a, Peru 197 \\
\hline Ecuador & Andes 3 & & & & $\zeta$-a, Ecuador 1, 3, 4, 7, 8, 13 \\
\hline Peru & Andes 4 & 0.983 & 4 & D & $\zeta$-a, Peru $196 ; \zeta$-g, Peru 65 \\
\hline Ecuador & Andes 4 & & & & $\zeta$-a, Ecuador 2, 5 \\
\hline Peru & Andes 5 & 0.977 & 2 & $\mathrm{G}$ & $\zeta$-a, Peru 88,100 \\
\hline Peru & Andes 6 & 0.982 & 2 & $\mathrm{G}$ & $\zeta$-a, Peru 92, 192 \\
\hline \multicolumn{6}{|c|}{ North America-U.S.A. } \\
\hline California & USA 1 & 0.976 & 4 & $\mathrm{E}$ & $\zeta-\mathrm{a}, 715006,715037,715038,715039$ \\
\hline California & USA 2 & 0.969 & 6 & $\mathrm{C}$ & $\zeta$-e, T29, CS339, $617,619,443$ \\
\hline Colorado & USA 2 & & & & $\alpha-\mathrm{e}, 63 \mathrm{CP} 55$ \\
\hline California & USA 3 & 0.987 & 4 & $\mathrm{C}$ & $\zeta$-а, 702596, 723064T; $\zeta$-e, CS283, 863 \\
\hline California & USA 4 & 0.988 & 3 & D & $\zeta-\mathrm{e}, \mathrm{CS} 118 \mathrm{~T}, 338$ \\
\hline New Mexico & USA 4 & & & & $\zeta-\mathrm{e}, \mathrm{S} . \mathrm{D}$. \\
\hline California & USA 5 & 0.974 & 2 & G & $\zeta-\mathrm{e}, \mathrm{CS} 22127$ \\
\hline Utah & USA 5 & & & & $\zeta-\mathrm{e}, 65 \mathrm{UT} 24$ \\
\hline California & USA 6 & - & 1 & $\mathrm{D}$ & $\zeta-\mathrm{a}, \mathrm{CS} 446$ \\
\hline California & USA 7 & 一 & 1 & $F$ & $\zeta-e, \operatorname{CS} 445$ \\
\hline \multirow[t]{4}{*}{ Madagascar } & $\underset{1}{\text { Madagascar }}$ & 0.970 & 3 & $\mathrm{C}$ & $\zeta-a$, Mad. $108,111_{\mathrm{a}}, 112$ \\
\hline & $\begin{array}{l}\text { Madagascar } \\
2\end{array}$ & 0.969 & 9 & D & $\begin{array}{l}\beta \text {-a, Mad. } 128 ; \zeta \text {-a, Mad. } 21,103_{\mathrm{a}} \\
109_{3}, 110,115,115_{3}, \mathrm{H}_{3}, \text { R.P. }\end{array}$ \\
\hline & $\begin{array}{l}\text { Madagascar } \\
3\end{array}$ & 一 & 1 & G & $\zeta$-a, Mad. 107 \\
\hline & $\begin{array}{l}\text { Madagascar } \\
\quad 4\end{array}$ & - & 1 & $\mathrm{G}$ & $\epsilon-\mathrm{a}$, Mad. $117_{\mathrm{a}}$ \\
\hline
\end{tabular}

${ }^{a}$ Average similarity matrix coefficient for the electropherotype.

${ }^{b}$ Number of cultures in electropherotype group.

c Biotypes corresponding to named varieties of $Y$. pestis are given in detail in Table 2.

d Actual origin Vietnam, isolate submitted with intentional error, correct designation was later revised to SEA 7 electropherotype. See text for details.

vole strain of $Y$. pestis antiqua (14), or an $\mathrm{A}-$, $Y$. pestoides altaica (16).

The electrophoretic and bacteriological groupings of the $161 Y$. pestis isolates studied are summarized in Table 2. Isolates have been listed by geographic origin, electropherotype, electropherotype group, and biotype. Actual relative optical density measurements of the nine protein bands utilized in defining the 58 electropherotypes were presented in Table 1.

To measure the effectiveness of our grouping scheme for various configurations containing a fixed number and size of subgroups of the 58 original electropherotypes of Fig. 4, we used a statistical procedure developed by John- son (11). The derivation of his test statistic, which makes no assumptions about the underlying distribution for the responses measured (here, the relative optical densities), is based on Euclidean distance as the measure of closeness in determining if responses are relatively homogenous within previously determined subgroups (here, those resulting from our similarity matrix methods). Assuming that the classification scheme yields results that are independent of any relationship among the specimens, we can determine the approximate probability ( $P$ value) that the test statistic has a value as extreme or more extreme than that observed. Mean similarity coefficients and $P$ 


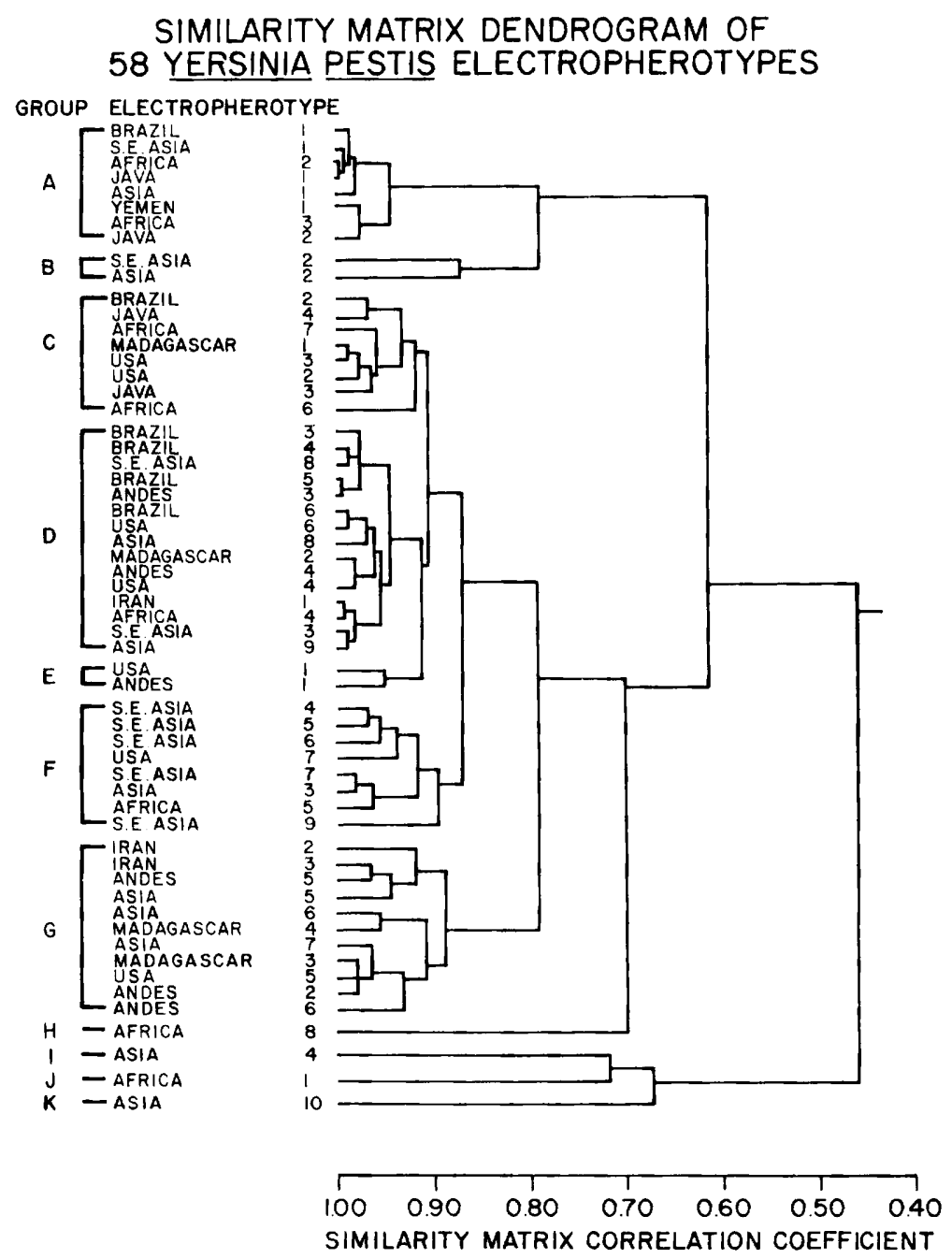

FIG. 4. Similarity matrix dendrogram of 58 Yersinia pestis electropherotypes. Major groups of electropherotypes, i.e., major protein variants, have been labeled $A$ to $K$.

values associated with the major electropherotype groups having sufficient numbers of cultures and subgroups for use in the test are: $\mathrm{A}, \mathbf{r}$ $=0.943, P=2 \times 10^{-20} ; \mathrm{C}, \mathrm{r}=0.916, P=9 \times$ $10^{-20} ; \mathrm{D}, \mathrm{r}=0.943, P=2 \times 10^{-239} ; \mathrm{E}, \mathrm{r}=$ $0.964, P=4 \times 10^{-4} ; \mathrm{F}, \mathrm{r}=0.893, P=2 \times$ $10^{-36}$; and $\mathrm{G}, \mathrm{r}=0.886, P=2 \times 10^{-39}$. The configuration composed of the seven subgroups $\mathrm{A}-\mathrm{G}$ results in $\mathrm{r}=0.914, P=2 \times 10^{-28}$. These extremely small probabilities not only emphasize the effectiveness of our particular grouping scheme but also make comparisons between this and other taxonomic schemes possible. This analysis can also be used to assess the differences between closely associated electropherotypes. Data obtained for electropherotypes closely related in the similarity matrix dendrogram (Fig. 4) are presented in Table 4. Only those electropherotypes having $P$ values equal to 0.01 or higher have been tabulated. For purposes of further discussion, we have also included the biotype information on the various $Y$. pestis isolates according to the scheme presented in Tables 1 and 2.

When the various groups of Table 4 are considered, the addition of the minimal biotype information supplied by the six biochemical tests allows all but a few isolates to be differentiated. Within the first couplet (Africa 2-Java 1 ), the $\zeta-\epsilon$ biotype, characteristic of the Java 1 electropherotype as well as all other Javanese isolates (see Table 2), is absent from the repre- 

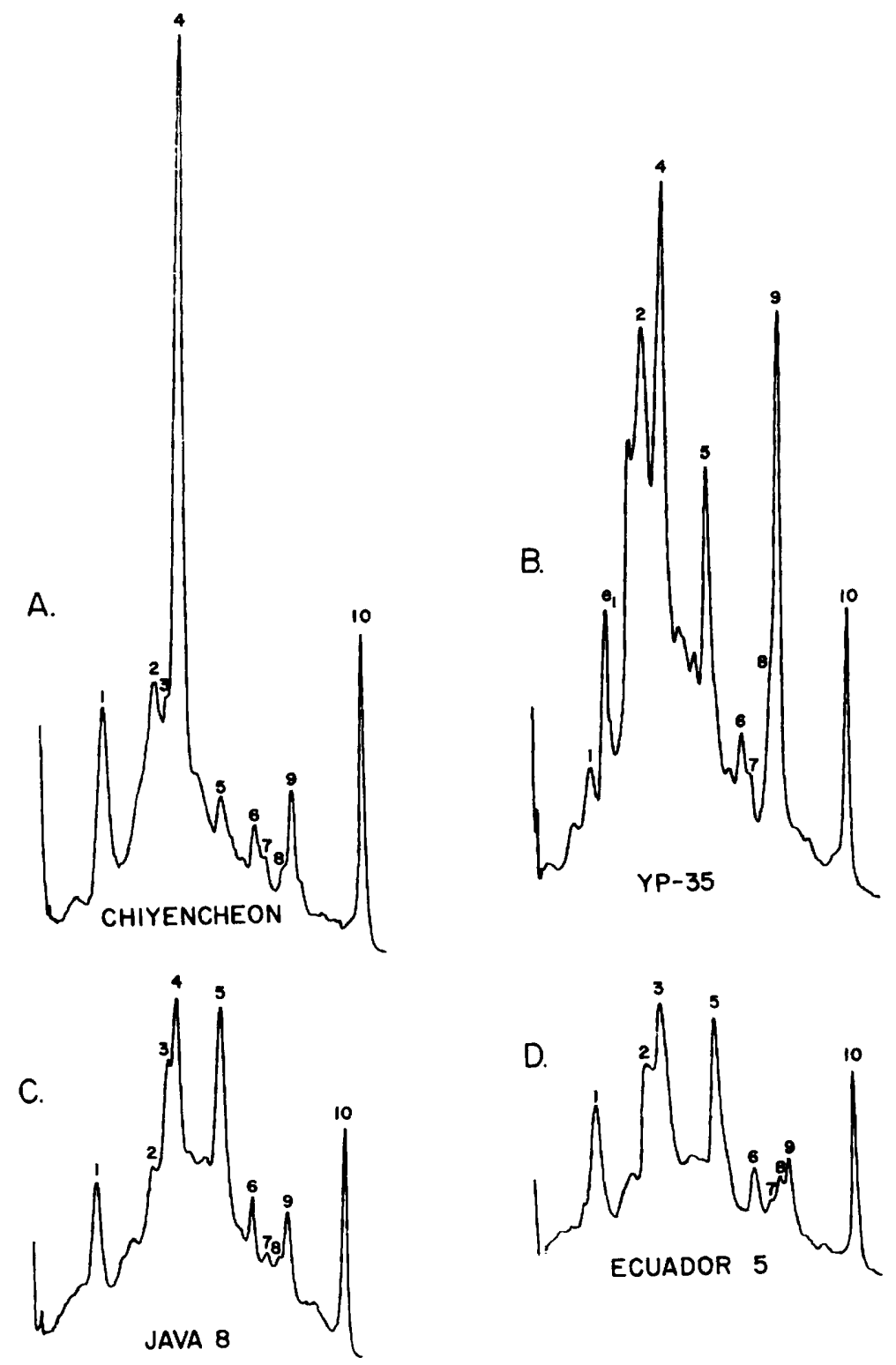

FIG. 5. Densitometric profiles of discontinuous acrylamide gel electropherograms of various Yersinia pestis isolates representing major protein variants.

sentatives of the Africa 2 electropherotype as well as the remainder of the African collection (Table 2 biotypes $\beta$-a, $\zeta$-a, -c, -g, -h). The second group, composed of the Yemen 1-Africa 3-Java 2 electropherotypes, is also separable by using biotype characteristics. The third group contains one Javanese isolate (Java 8) and one Brazilian isolate (Exú 1), which are not separable by the criteria presented. These two isolates, however, have been shown to differ with regard to asparagine dependence in our previous report (10). The fourth group, Madagascar 1-USA 3, contains five isolates not separable by biotype-electropherotype groupings. The isolates involved are Madagascar 108, 111 a 112 and the California isolates 702596, 723064T, all five of which possess the biotype $\zeta$-a $(\mathrm{G}-\mathrm{N}+$ RHA - A + M+ X+). The members of the fifth couplet, Brazil 4-SEA 8, are glycerol-positive Nepalese and glycerol-negative Brazilian iso- 

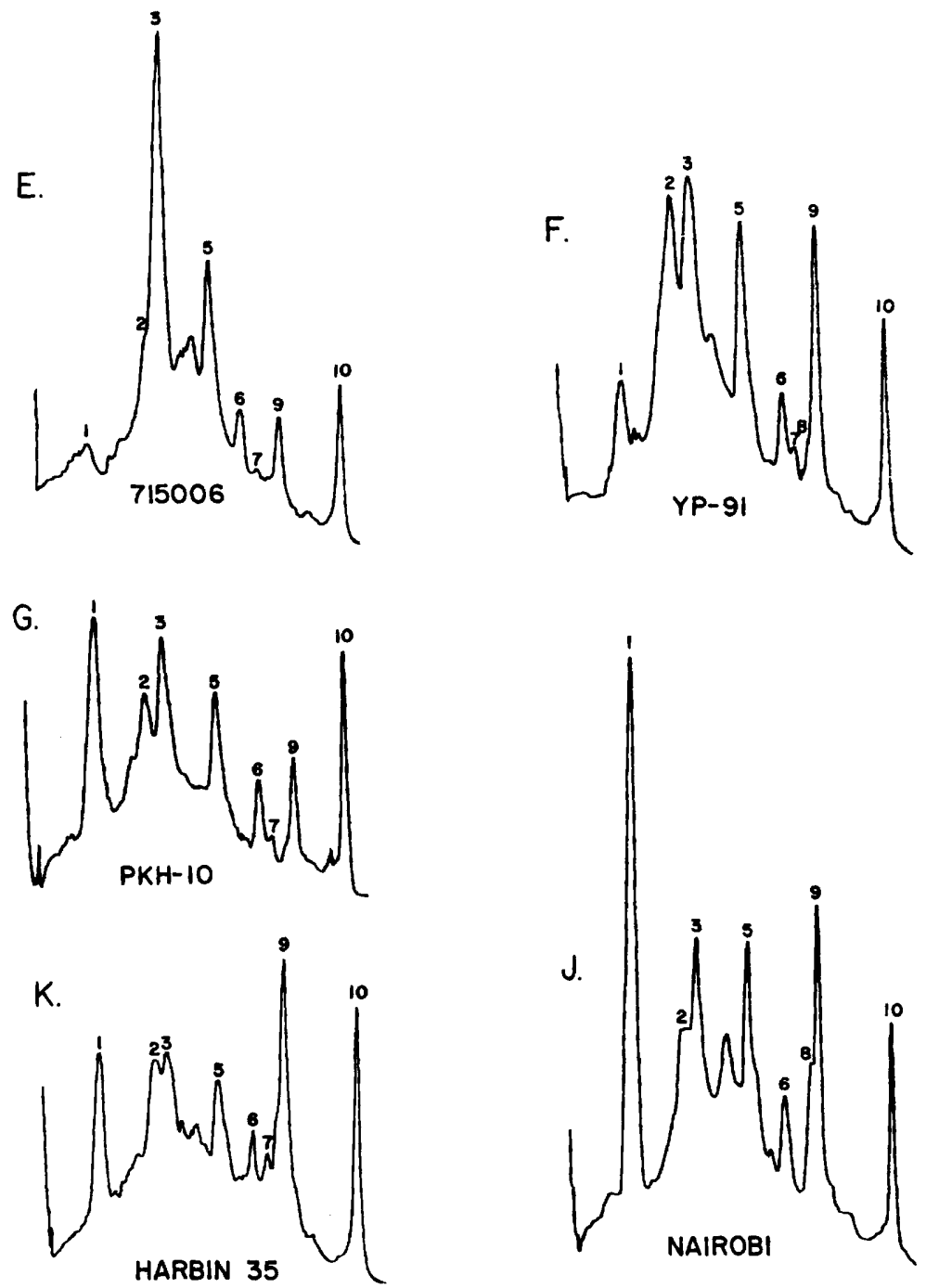

FiG. 5.-continued

lates. Couplet 6, from Peru and Brazil, is separable on the basis of arabinose utilization. The seventh couplet, labeled SEA-7-Asia 3, has a $P$ value equal to 0.22 . The isolate YP-91, originally received and analyzed together with the Asian isolants from the U.S.S.R. and Manchuria, was found to be electrophoretically distinct from the remainder of the Asian isolates. The electropherotype (Asia 3) was found to be similar to electropherotype SEA-7, composed of a number of Burmese isolates of various biotypes. Additionally, the biotype of isolate YP-91, $(\epsilon-a), \mathrm{G}-\mathrm{N}+\mathrm{RHA}+\mathrm{A}+\mathrm{M}+$ $\mathrm{X}+$, consisted of an unusual rhamnose-positive variant of $Y$. pestis orientalis identical to one isolate in the Burmese electropherotype. At this point we contacted the colleague originally submitting the specimen and were informed that the mislabeling was an intentional test of the analytical process by a single blind experiment. Isolate YP-91 had originated in Vietnam, and, on reanalysis of its electrophoretic data in the SEA group of isolates, we placed it within the SEA 7 electropherotype $(r=0.975)$, thus reducing the number of electropherotypes to 57.

\section{DISCUSSION}

The sample of $161 Y$. pestis isolates from nine geographic regions were assembled into 57 electropherotypes, representing roughly 11 major protein variants on a worldwide basis. 
TABLE 3. Biotype coding of $Y$. pestis isolates by three major and three minor biochemical tests

\begin{tabular}{|c|c|c|c|c|c|c|c|c|}
\hline \multicolumn{5}{|c|}{ Major biotypes } & \multicolumn{4}{|c|}{ Minor biotypes } \\
\hline \multirow{2}{*}{ Code } & \multirow{2}{*}{ Subspecific designation } & \multicolumn{3}{|c|}{ Biochemical tests $^{n}$} & \multirow{2}{*}{ Code } & \multicolumn{3}{|c|}{ Biochemical tests $^{a}$} \\
\hline & & G & $\mathbf{N}$ & RHA & & A & $\mathbf{M}$ & $\mathrm{x}$ \\
\hline$\alpha$ & $\begin{array}{l}Y . p . \text { antiqua }(\text { vole }) \\
(Y . \text { pestoides altaica })\end{array}$ & + & + & + & a & + & + & + \\
\hline$\beta$ & $Y . p$. antiqua & + & + & - & $\mathbf{b}$ & + & + & - \\
\hline$\gamma$ & $\begin{array}{l}Y . p . \text { mediaevalis (vole) } \\
\quad(Y . \text { pestoides parvocaucasica) }\end{array}$ & + & - & + & c & + & - & + \\
\hline$\delta$ & $Y . p$. mediaevalis & + & - & - & d & + & - & - \\
\hline$\epsilon$ & $\begin{array}{l}Y . p . \text { orientalis } \\
\text { (unnamed variant) }\end{array}$ & - & + & + & e & - & + & + \\
\hline$\zeta$ & $Y . p$. orientalis & - & + & - & f & - & + & - \\
\hline$\eta$ & $\begin{array}{l}Y . \text { pestis } \\
\text { (unnamed variant) }\end{array}$ & - & - & + & g & - & - & + \\
\hline$\theta$ & $\begin{array}{l}Y \text {. pestis } \\
\quad \text { (unnamed variant) }\end{array}$ & - & - & - & $\mathbf{h}$ & - & - & - \\
\hline
\end{tabular}

${ }^{a}$ Abbreviations: G, glycerol; N, nitrate reduction; RHA, rhamnose; $\mathrm{A}$, arabinose; $\mathrm{M}$, maltose; $\mathrm{X}, \mathrm{xylose}$.

TABLE 4. Statistical and biochemical comparison of closely related electropherotypes

\begin{tabular}{|c|c|c|c|c|}
\hline Electropherotypes & $\mathbf{r}^{\prime}$ & $P$ value & No. of isolates & Biotypes (no. and kind) \\
\hline Africa 2 & 0.998 & 0.035 & 5 & $\begin{array}{l}1, \zeta-\mathrm{h} ; 1, \zeta-\mathrm{g} ; \\
1, \zeta-\mathrm{c} ; 2, \zeta-\mathrm{a}\end{array}$ \\
\hline Java 1 & & & 13 & $13, \zeta-\mathrm{e}$ \\
\hline $\begin{array}{l}\text { Yemen } 1 \\
\text { Africa } 3 \\
\text { Java } 2\end{array}$ & 0.976 & 0.094 & $\begin{array}{l}2 \\
1 \\
2\end{array}$ & $\begin{array}{l}2, \delta \text {-a } \\
\zeta-\mathrm{c} \\
2, \zeta-\mathrm{e}\end{array}$ \\
\hline $\begin{array}{l}\text { Brazil } 2 \\
\text { Java } 4\end{array}$ & 0.974 & $-^{b}$ & $\begin{array}{l}2 \\
1\end{array}$ & $\begin{array}{l}1, \zeta \text {-a; } 1, \zeta-\mathrm{e} \\
1, \zeta-\mathrm{e}\end{array}$ \\
\hline $\begin{array}{l}\text { Madagascar } 1 \\
\text { USA } 3\end{array}$ & 0.990 & 0.094 & $\begin{array}{l}3 \\
4\end{array}$ & $\begin{array}{l}3, \zeta-\mathrm{a} \\
2, \zeta-\mathrm{a} ; 2, \zeta-\mathrm{e}\end{array}$ \\
\hline $\begin{array}{l}\text { Brazil } 4 \\
\text { SEA } 8\end{array}$ & 0.986 & 0.27 & $\begin{array}{l}2 \\
2\end{array}$ & $\begin{array}{l}1, \zeta \text {-e; } 1, \zeta \text {-g } \\
1, \delta \text {-g; } 1, \delta \text {-h }\end{array}$ \\
\hline $\begin{array}{l}\text { Brazil } 5 \\
\text { Andes } 3\end{array}$ & 0.996 & 0.013 & $\begin{array}{l}3 \\
8\end{array}$ & $\begin{array}{l}1, \zeta-\mathrm{e} ; 2, \zeta-\mathrm{g} \\
7, \zeta-\mathrm{a} ; 1, \zeta-\mathrm{e}\end{array}$ \\
\hline SEA 7 & 0.975 & 0.22 & 5 & $\begin{array}{l}3, \zeta-\mathrm{a} ; 1, \theta-\mathrm{a} \\
\quad 1, \epsilon-\mathrm{a}\end{array}$ \\
\hline Asia $3^{c}$ & & & 1 & $1, \epsilon-\mathbf{a}$ \\
\hline
\end{tabular}

${ }^{a}$ Average similarity matrix coefficient.

${ }^{b}$ Numbers of isolants insufficient for statistical analysis of group.

c Originally labeled as Asian isolate, actual origin Vietnam.

Only seven of the $Y$. pestis isolates studied could not be characterized as members of unique geographical groups on the basis of the electropherotype and the minimal biotype characteristics used. These seven isolates possibly could be successfully separated by adding other biochemical tests. The use of asparagine dependence assay has previously shown that two of the seven, Java 8-Exú 1, differ in this regard. Additionally, the incident of the mislabeled YP-91 isolate demonstrates that at least in one case a deceptive submission could be successfully associated with an electropherotype characteristic of its geographic source.

Although this consistency is of undoubted value in assessing the potential of electrophoretic techniques for the analysis of $Y$. pestis isolates, one other source of potential variation 
should be noted. The KIM-10 series of selected biochemical variants was analyzed to assess the importance of biochemical variation relative to electrophoretic characteristics. Analysis of the group yielded an average similarity coefficient of 0.967 , a value within our estimated range for electropherotypes (i.e., $\mathrm{r} \geq 0.963$ ). The isolate KIM-10, however, originated 10 years ago as a subculture of the Pasteur Institute isolate, PKH-10. The PKH-10 culture was freeze-dried shortly after the original KIM-10 subculture had been taken. Comparison of the results obtained for isolate KIM-10 and our subculture of the original PKH-10 isolate is of interest in regard to possible changes caused by differing maintenance regimes. Our comparison of KIM-10 and PKH-10 revealed a prominent difference in the amplitude of protein band number 1 . This difference cannot be readily explained, owing to lack of information pertaining to the history of animal passage and subculturing of the KIM-10 stock. From the uniform results obtained with the KIM-10 series, however, it appears that, once such variation in disc gel characteristics occurs, it may constitute a stable characteristic under conditions prevailing during the selection of biochemically variant subcultures.

The most extensive early studies of geographic distribution of $Y$. pestis varieties are those of Devignat (5), who defined three major varieties, or races, based on glycerine fermentation and nitrate reduction. He considered the antiqua variety $(\mathrm{G}+, \mathrm{N}+)$ to be the most likely candidate of the ancestral variety. Originating in Central Asia and spreading in biblical times to Africa, this variety was reported in Central Africa, Manchuria, and Mongolia. Our, sample includes $\mathrm{G}+, \mathrm{N}+$ variants from Nepal, Manchuria, the U.S.S.R. (unknown localities), Kenya, Madagascar, and Iran. Variety mediaevalis $(\mathrm{G}+, \mathrm{N}-)$ was assumed to have originated in the region of the Caspian Sea and to have spread throughout Europe during the pandemic of the medieval period; it is now entrenched in Iranian Kurdistan and Southeast Russia. $\mathrm{G}+, \mathrm{N}-$ variants in our sample are from Yemen, the U.S.S.R., Nepal, and Iran. Variety orientalis $(\mathrm{G}-, \mathrm{N}+)$, responsible for the present pandemic, originated in the Yunnan Province of China, was spread from southeast Asia throughout the modern world, and is presently entrenched in rodent foci in North and South America, Africa, Madagascar, Southeast Asia, India, Indonesia, and Manchuria. With the exception of Manchuria, this distribution corresponds to that obtained for $(G-, N+)$ isolates in our sample.
The last major variant of the plague organism was described by Levi and his co-workers in 1961 (14). This consists of rhamnose-positive variants of glycerol-positive $Y$. pestis. Martinevskij (16) has recently reviewed systems of nomenclature for $Y$. pestis. In this review he suggested two species, $Y$. pestis and $Y$. pestoides. $Y$. pestis is composed of three varieties corresponding to Devignat's antiqua ( $Y$. pestis mediaasiatica montana), mediaevalis ( $Y$. pestis mediaasiatica deserta), and orientalis ( $Y$. pestis oceanica). Y. pestoides, Levi's rhamnose-positive strains, are separated into two ecotypes corresponding to two varieties secured from the Caucasus and the Altai, respectively.

Classification of $Y$. pestis isolates into biotypes and major protein variants or electropherotype groups yields certain conclusions corresponding in general to Devignat's original hypothesis (5). First, group A electropherotypes of $Y$. pestis orientalis (Table 2) are found in Java, Brazil, South Africa, India, and Burma. Second, the Kenya isolate, Nairobi (electropherotype $J$ biotype $\beta-a$ ), shows a degree of similarity to the Manchurian isolate, Harbin $35(\mathrm{~K}, \beta-\mathrm{a})$. Third, variants present in subgroup $E$ of the dendrogram are from discrete, geographically limited foci in the San Francisco Bay area of California and in Apollo, Bolivia, both electropherotypes being dissimilar from others present in their geographically contiguous areas. Fourth, examination of subgrouping indicates a trend toward similarity between electropherotypes from the Andean region, Madagascar, and the United States. The distribution of the group A, Y. pestis orientalis $(\mathrm{G}-, \mathrm{N}+$ ) electropherotypes corresponds with one of Devignat's routes of worldwide dispersion of $Y$. pestis orientalis during the present pandemic. Devignat gives this route as Hong Kong-India-Madagascar and South Africa-Brazil. With the exception of our Madagascar samples, this is the same as the distribution of the group A, Y. pestis orientalis electropherotypes. Another route of dispersal was given as Hong Kong to Hawaii to the west coasts of North and South America. These areas possess $Y$. pestis strains with electrophoretic characteristics resembling each other and, in addition to Vietnam and Madagascar, are the only areas sampled in which group A electropherotypes are absent.

No overall relationship between electropherotype groupings and major biotypes is evident in Table 2. The SEA 8 electropherotype contains biotypes $\delta$ and $\beta$ corresponding to $Y$. pestis mediaevalis and $Y$. pestis antiqua. Similarly, the Madagascar 2 electropherotype contains 
$\zeta$ and $\beta$ biotypes corresponding to $Y$. pestis orientalis and $Y$. pestis antiqua. Additionally, the major electropherotype groups contain a variety of major biotypes. Group A, for instance, contains $\alpha, \delta$, and $\zeta$ biotypes corresponding to $Y$. pestis antiqua ( $Y$. pestoides), $Y$. pestis mediaevalis, and $Y$. pestis orientalis. In spite of this lack of overall correspondence between biotype and electropherotype, it appears that strains isolated within one limited geographic region, particularly isolates from geographically and epidemiologically limited foci, show similarities to each other and can be differentiated from similar isolates or electropherotypes found in other regions. At present, we are unable to explain the origin of these differences. Apparently, the factors responsible for major protein variants such as the group A $Y$. pestis isolates of this report may be fixed, conservative characters, even under the conditions prevailing when the bacterium is introduced into new geographic regions. After the original introduction, other quantitative changes in protein constituents may occur and remain as moderate to long-term characteristics of plague isolates found in certain plague foci.

Whether any selective advantage is involved in these variations is as yet unsettled. Only one protein of the nine studied has been identified. The band 4 protein has been isolated and identified as subfraction $B$ of the murine toxin of $Y$. pestis (7).

Electrophoretic techniques have been used for phenotypic differentiation of small rodent populations (15), for genetic analysis of laboratory strains of Tetrahymena (2), and for comparison of mycobacterial antigens (17). We believe that the qualitative differences detected between the major protein variants of $Y$. pestis may also be an indication of significant phenotypic differences. The precise genetic status of the variants, however, is not known. Each area in which major electropherotype groups are found also has other protein variants extant in immediate or contiguous geographic areas. For this reason, the electrophoretic variants probably do not represent subspecific taxonomic traits. Classification by electropherotypes may, however, represent a potential method for epidemiological analysis of closely related isolates. That this classification is probably not correlated with biochemical traits used in the bacteriological classification of $Y$. pestis may be an advantage in this context, since it should allow a greater flexibility in yielding an individualized characterization of the isolates examined. The numbers of isolates examined from any of the geographic regions covered in this study are too few, however, to draw any final conclusions with regard to the ultimate validity of electrophoretic analysis for epizootiological studies of the geographic distribution of the various electropherotypes within specific localities. We are of the opinion, however, that this should be a matter of concern in later studies of this technique.

\section{ACKNOWLEDGMENTS}

We thank numerous colleagues for their generous help and assistance in supplying materials for these studies. In particular, these colleagues are the late $M$. Baltazard, who supplied the U.S.S.R. rhamnose-positive variants; $M$. Isaacson, who provided the South African isolates; A. Wake, who provided the Manchurian isolates; M. Bahmanyar, who supplied isolates from Yemen, Iran, India, Burma, and Brazil; E. Antezana, who provided the isolates from Peru and Ecuador; and Dan Cavanaugh and John Marshall, who supplied the isolates from Burma and Vietnam.

\section{REPRINT REQUESTS}

Address reprint requests to: Dr. T. J. Quan, Dept. of Health, Education, and Welfare, Bureau of Laboratories, Vector-Borne Diseases Division, P.O. Box 2087, Fort Collins, Colo. 80522.

\section{LITERATURE CITED}

1. Baltazard, M., D. H. S. Davis, R. Devignat, G. Girard, M. A. Gohar, L. Kartman, K. F. Meyer, M. T. Parker, R. Pollitzer, F. M. Prince, S. F. Quan, and P. Wagle. 1956. Recommended laboratory methods for the diagnosis of plague. Bull. W.H.O. 14:457-509.

2. Borden, D., G. S. Whitt, and D. L. Nanney. 1973. Isozymic heterogeneity in Tetrahymena strains. Science 181:279.

3. Center for Disease Control. August 14, 1968. Report on the investigation and control of plague in Central Java, Indonesia. Atlanta, Georgia.

4. Cowan, S. T., and K. J. Steele. 1965. Manual for the identification of medical bacteria. Cambridge University Press, Cambridge.

5. Devignat, R. 1953. La Peste antique du Congo belge dans le cadre de l'Histoire et de la Geographie. Inst. Royal Colonial Belge, Mem. 23:1-48.

6. Dowdle, W. R., M. T. Coleman, E. C. Hall, and V. Knez. 1969. Properties of the Hong Kong Influenza virus. 2. Antigenic relationship of the Hong Kong virus haemagglutinin to that of other human Influenza A viruses. Bull. W.H.O. 41:419-424.

7. Hillam, R. P., R. P. Tengerdy, and G. L. Brown. 1974 Local antibody production against the murine toxin of Yersinia pestis in a golf ball-induced granuloma. Infect. Immun. 10:458-463.

8. Hudson, B. W., and M. I. Goldenberg. 1970. Isolation of Yersinia pestis of unusual protein content obtained from Central Java. Bull. W.H.O. 43:917-919.

9. Hudson, B. W., and T. J. Quan. 1975. Electrophoretic studies of the Yersiniae. Am. J. Trop. Med. Hyg. 24:968-973.

10. Hudson, B. W., T. J. Quan, V. R. Sites, and J. D. Marshall. 1973. An electrophoretic and bacteriologic study of Yersinia pestis isolates from Central Java, Asia and the Western Hemisphere. Am. J. Trop. Med. Hyg. 22;642-653.

11. Johnson, E. S. 1973. A multi-response permutation test 
for measuring the effectiveness of $a$ priori classification. Univ. Microfilms, Ann Arbor, no. 34/09-B, 1974, Microfilm no. 74-5423, VII:1-72.

12. Lee, A. M. 1968 . Numerical taxonomy and Influenza $B$ virus. Nature (London) 217:620-622.

13. Lee, A. M., and N. M. Tauraso. 1968. A method for the formulation of Influenza virus vaccine using numerical taxonomy. Bull. W.H.O. 39:261-270.

14. Levi, M. I., Yu. V. Kanatov, L. A. Sagatovskaya, and Ye. A. Kanatova. 1961. A new variety of the plague microbe. Trudy Rostovskogo-na-Donu Gosudarstvennogo Nauchno-issledovatel'skogo Protivo- chumnogo Inst. 18:3-23.

15. Marsh, R. E., W. E. Howard, and H. Bongardt. 1969. Some serum transferrins in California ground squirrels (Spermophilus). J. Mammal. 50:649-652.

16. Martinevskij, I. L. 1967. Taxonomie du genre Yersinia et nomenclature de ses representants. Int. Symp. Pseudotuberculosis, Paris 1967. Symp. Series Immunobiol. Standard. 9:361-368.

17. Roberts, D. R., G. L. Wright, L. F. Affronti, and M. Reich. 1972. Characterization and comparison of mycobacterial antigens by two-dimensional immunoelectrophoresis. Infect. Immun. 6:564-573. 